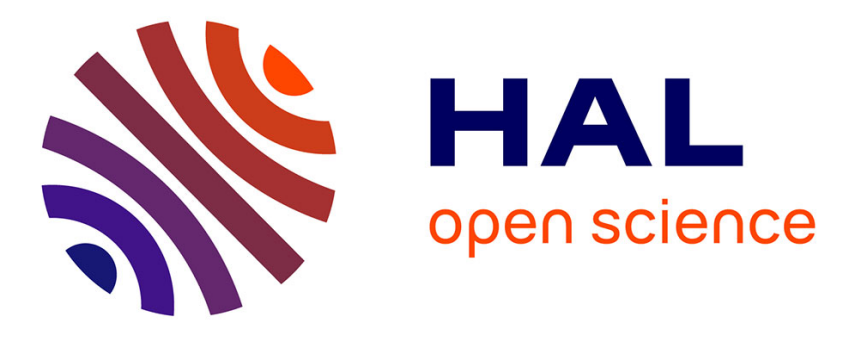

\title{
Numerical study of long waves in ferromagnetic media
}

David Sanchez

\section{To cite this version:}

David Sanchez. Numerical study of long waves in ferromagnetic media. Numerical Methods for Partial Differential Equations, 2006, 22 (5), pp.1127 - 1148. 10.1002/num.20142 . hal-01872056

\section{HAL Id: hal-01872056 https://hal.science/hal-01872056}

Submitted on 11 Sep 2018

HAL is a multi-disciplinary open access archive for the deposit and dissemination of scientific research documents, whether they are published or not. The documents may come from teaching and research institutions in France or abroad, or from public or private research centers.
L'archive ouverte pluridisciplinaire HAL, est destinée au dépôt et à la diffusion de documents scientifiques de niveau recherche, publiés ou non, émanant des établissements d'enseignement et de recherche français ou étrangers, des laboratoires publics ou privés. 


\title{
Numerical study of long waves in ferromagnetic media.
}

\author{
DAVID SANCHEZ \\ Mathématiques Appliquées de Bordeaux, UMR 5466, Université Bordeaux 1, \\ 351 Cours de la Libération, 33405 Talence cedex, France. \\ e-mail: david.sanchez@math.u-bordeaux1.fr
}

\begin{abstract}
We numerically study small bidimensional perturbations of electromagnetic waves in a saturated ferromagnetic media. We develop and compare three schemes and highlight some properties of the system.
\end{abstract}

Keywords. Micromagnetics, ferromagnets, Maxwell equations, Landau-Lifshitz equation, numerical approximation.

\section{Introduction}

The state of a ferromagnet is described by the magnetization vector $M$ whose dynamic equation was first given by Landau and Lifshitz [6] and later in an equivalent form by Gilbert [4]:

$$
\frac{\partial M}{\partial t}=-M \wedge \mathcal{H}-\gamma M \wedge(M \wedge \mathcal{H}) \quad \text { in } \Omega,
$$

where $\gamma$ is a dimensionless damping coefficient and $\mathcal{H}$ the effective field is given by:

$$
\mathcal{H}=\eta \Delta M+\varphi(M)+H,
$$

where $\eta \Delta M$ is the exchange term, $\varphi(M)$ is the anisotropy field and $H$ the magnetic field. We assume in the following that there is no anisotropy field and that the exchange coefficient $\eta$ is small enough. This last assumption implies that the influence of the exchange term is negligible far from the boundary of the domain (see [2]). We moreover only look at the behaviour in the core of the ferromagnet thus working in the whole space instead of working in a bounded domain:

$$
\frac{\partial M}{\partial t}=-M \wedge H-\gamma M \wedge(M \wedge H) \quad \text { in } \mathbb{R}^{3},
$$

We can note that the magnitude $|M|$ of $M$ is an invariant of this equation. The equation is completed by the Maxwell equations for the electromagnetic field:

$$
\left\{\begin{array}{l}
\frac{\partial(H+M)}{\partial t}-\nabla \wedge E=0 \quad \text { in } \mathbb{R}^{3}, \\
\frac{\partial E}{\partial t}+\nabla \wedge H=0 \quad \text { in } \mathbb{R}^{3},
\end{array}\right.
$$

where we assume that there is no electric polarization $(D=E)$ and where the equations are in dimensionless form and the coefficients have been set equal to one.

The system of equations (1.2)-(1.3) admits a family of constant solutions,

$$
(M, H, E)_{\alpha}=\left(M_{0}, \alpha^{-1} M_{0}, 0\right), \quad \alpha>0,
$$

AMS Subject Classifications: 35Q60, 65M06, 65M99, 82D40. 
where $M_{0}$ is an arbitrary vector in $\mathbb{R}^{3}$ and $\alpha>0$ assures that the steady solution is stable. We may assume without restriction that $\left|M_{0}\right|=1$.

We are interested in the spatio-temporal evolution of long-waves and transverse perturbations of such solutions. Leblond and Manna observe that these perturbations behave like travelling and stationary waves for which diffraction effects occur for long times.

These perturbations are measured in terms of an arbitrarily small positive parameter $\varepsilon$ and have the form:

$$
\begin{aligned}
& M(t, x, y)=M_{0}+\varepsilon^{s} \widetilde{M}(\widetilde{t}, \tau, \widetilde{x}, \widetilde{y}), \\
& H(t, x, y)=\alpha^{-1} M_{0}+\varepsilon^{s} \widetilde{H}(\widetilde{t}, \tau, \widetilde{x}, \widetilde{y}), \\
& E(t, x, y)=\varepsilon^{s} \widetilde{E}(\widetilde{t}, \tau, \widetilde{x}, \widetilde{y})
\end{aligned}
$$

where $\widetilde{M}, \widetilde{H}$ and $\widetilde{E}$ are $O(1)$ as $\varepsilon$ goes to 0 , and:

$$
\widetilde{x}=\varepsilon^{n} x, \quad \widetilde{y}=\varepsilon^{p} y, \quad \widetilde{t}=\varepsilon^{n} t, \quad \tau=\varepsilon^{r} t .
$$

Following H. Leblond $[8,7]$ we now set the characteristic sizes of the problem. We assume here that we are in the weakly non-linear case, i.e. $s>0$. We need this assumption to perform NLS-type expansions. We look for solutions propagating in the $x$-direction - still unknown which explains the same scaling $\varepsilon^{n}$ for $\widetilde{x}$ and $\widetilde{t}$; the case of long or low-frequency waves we consider corresponds to the case $n>0$. We are looking for solutions for which the dispersion and the non-linear effects occur at the same long time scale. As the parameter $n$ sets the time to which the dispersion effects occur, $s$ the intensity of the non-linear effects and thus the time where they occur, we choose them such that these effects occur at the same long time (set by $r)$. Here,

$$
r=4, \quad n=2, \quad s=2 .
$$

We also want to study transversal deformations of these quasi-plane waves. This case corresponds to the scaling $\widetilde{y}=\varepsilon^{p} y$ with $p>2$. There is a dominant diffraction effect that occurs for the smallest value of $p$ for which we can "solve" the equation, which is here $p=3$. If we take $2<p<3$, the diffraction effect prevails over the dispersion and prevents us to observe the non-linear effects. On the contrary, if $p>3$ the diffraction is negligible.

Although many numerical studies exist on the Landau-Lifshitz-Maxwell equations (Halpern and Labbé [5], Monk and Vacus [9]) we develop here numerical schemes designed to study specifically the behaviour of the perturbation on long times and expect to observe the nonlinear and diffractive phenomena. Since these effects occur for time of order $1 / \varepsilon^{2}$ we forget the long time variable $\tau$ but perform the study on the required times in order to observe the effects:

If $(M, H, E)$ is a solution of equations (1.2)-(1.3) then $\widetilde{M}, \widetilde{H}$ and $\widetilde{E}$ have to fulfill:

$$
\begin{aligned}
& \varepsilon^{2} \partial_{\tilde{t}} \widetilde{M}=-M_{0} \wedge \widetilde{H}+\alpha^{-1} M_{0} \wedge \widetilde{M}-\varepsilon^{2} \widetilde{M} \wedge \widetilde{H}-\gamma\left[M_{0} \wedge\left(M_{0} \wedge \widetilde{H}\right)\right. \\
& -\alpha^{-1} M_{0} \wedge\left(M_{0} \wedge \widetilde{M}\right)+\varepsilon^{2} M_{0} \wedge(\widetilde{M} \wedge \widetilde{H})+\varepsilon^{2} \widetilde{M} \wedge\left(M_{0} \wedge \widetilde{H}\right) \\
& \left.-\varepsilon^{2} \alpha^{-1} \widetilde{M} \wedge\left(M_{0} \wedge \widetilde{M}\right)+\varepsilon^{4} \widetilde{M} \wedge(\widetilde{M} \wedge \widetilde{H})\right] \text {, } \\
& \left\{\begin{array}{l}
\varepsilon^{2} \partial_{t} \widetilde{H}-\varepsilon^{2} \widetilde{\nabla}_{\widetilde{x}} \wedge \widetilde{E}-\varepsilon^{3} \widetilde{\nabla}_{\widetilde{y}} \wedge \widetilde{E}=-\varepsilon^{2} \partial_{\widetilde{t}} \widetilde{M}-\varepsilon^{4} \partial_{\tau} \widetilde{M}, \\
\varepsilon^{2} \partial_{\tilde{t}} \widetilde{E}+\varepsilon^{2} \widetilde{\nabla}_{\widetilde{x}} \wedge \widetilde{H}+\varepsilon^{3} \widetilde{\nabla}_{\widetilde{y}} \wedge \widetilde{H}=0,
\end{array}\right.
\end{aligned}
$$


We are interested in solutions of Eqs. (1.2)-(1.3) that describe a travelling wave propagating in the direction $\widetilde{k}$ with transversal perturbations in the direction $\widetilde{l}$ where $\widetilde{k}$ and $\widetilde{l}$ are fixed unit vectors. According to [8] and [11] we assume:

$$
\widetilde{k} \wedge M_{0} \neq 0, \quad \widetilde{k} \cdot \widetilde{l}=0, \quad \tilde{l} \cdot M_{0}=0 .
$$

By assuming that $\widetilde{x}$ is the coordinate in the $\widetilde{k}$-direction and $\widetilde{y}$ in the $\widetilde{l}$-direction we may perform the substitutions:

$$
\widetilde{\nabla}_{\widetilde{x}}=\widetilde{k} \partial_{\widetilde{x}} \text { and } \quad \widetilde{\nabla}_{\widetilde{y}}=\widetilde{l}_{\widetilde{y}} .
$$

Henceforth we omit the tilde, so the equations to be considered are:

$$
\begin{aligned}
\partial_{t} M= & \frac{1}{\varepsilon^{2}}\left(-M_{0} \wedge H+\alpha^{-1} M_{0} \wedge M\right)-M \wedge H-\frac{\gamma}{\varepsilon^{2}}\left[M_{0} \wedge\left(M_{0} \wedge H\right)\right. \\
& \left.-\alpha^{-1} M_{0} \wedge\left(M_{0} \wedge M\right)\right]-\gamma\left[M_{0} \wedge(M \wedge H)+M \wedge\left(M_{0} \wedge H\right)\right. \\
& \left.-\alpha^{-1} M \wedge\left(M_{0} \wedge M\right)+\varepsilon^{2} M \wedge(M \wedge H)\right], \\
\partial_{t} H-k \wedge & \partial_{x} E-\varepsilon l \wedge \partial_{y} E=-\partial_{t} M, \\
\partial_{t} E+k \wedge & \partial_{x} H+\varepsilon l \wedge \partial_{y} H=0 .
\end{aligned}
$$

This system fulfills the following energy estimates (see Section 2):

Theorem 1.1. Let $(H, E, M)$ a regular solution of (1.6)-(1.7)-(1.8) with the initial data $\left(\widetilde{E}_{0}, \widetilde{H}_{0}, \widetilde{M}_{0}\right)$ and $T>0$ their maximum existence time. The solution then satisfies for all $0 \leq t<T$,

$$
\begin{aligned}
& \frac{1}{2}\left(\|H\|_{\mathbb{L}^{2}\left(\mathbb{R}^{2}\right)}^{2}+\|E\|_{\mathbb{L}^{2}\left(\mathbb{R}^{2}\right)}^{2}+\alpha^{-1}\|M\|_{\mathbb{L}^{2}\left(\mathbb{R}^{2}\right)}^{2}\right)(t) \\
& +\gamma \int_{0}^{t}\left\|\frac{1}{\varepsilon} M_{0} \wedge H+\varepsilon M \wedge H-\frac{1}{\varepsilon \alpha} M_{0} \wedge M\right\|_{\mathbb{L}^{2}\left(\mathbb{R}^{2}\right)}^{2} d t \\
= & \frac{1}{2}\left(\left\|\widetilde{H}_{0}\right\|_{\mathbb{L}^{2}\left(\mathbb{R}^{2}\right)}^{2}+\left\|\widetilde{E}_{0}\right\|_{\mathbb{L}^{2}\left(\mathbb{R}^{2}\right)}^{2}+\alpha^{-1}\left\|\widetilde{M}_{0}\right\|_{\mathbb{L}^{2}\left(\mathbb{R}^{2}\right)}^{2}\right) .
\end{aligned}
$$

We then develop in Section 3 some numerical schemes to solve on a long time scale the equations fulfilled by the perturbation. We obtain a semi-implicit scheme that fulfills Eq. (1.9):

$$
\begin{aligned}
& \frac{1}{2}\left(\left\|H^{n+1}\right\|^{2}+\left\|E^{n+1}\right\|^{2}+\alpha^{-1}\left\|M^{n+1}\right\|^{2}\right) \\
& +d t \gamma\left\|\frac{1}{\varepsilon} M_{0} \wedge H^{n+1 / 2}+\varepsilon M^{n} \wedge H^{n+1 / 2}-\frac{1}{\varepsilon \alpha} M_{0} \wedge M^{n+1 / 2}\right\|^{2} \\
= & \frac{1}{2}\left(\left\|H^{n}\right\|^{2}+\left\|E^{n}\right\|^{2}+\alpha^{-1}\left\|M^{n}\right\|^{2}\right),
\end{aligned}
$$

where $V^{n+1 / 2}=\frac{V^{n+1}+V^{n}}{2}$ and $\|\cdot\|$ is the $\mathbb{L}^{2}$-norm on the discretized spatial domain.

Unfortunately this scheme does not preserve the norm of the full magnetization $M_{0}+\varepsilon^{2} M$ neither that the divergence $\operatorname{div}(H+M)$ does not vary in the course of time. This scheme also requires at each step the inversion of a $18 N_{a} N_{b} \times 18 N_{a} N_{b}$ matrix (where $N_{a}$ and $N_{b}$ are the number of points of discretization).

Using a splitting in time and the Yee scheme to solve the Maxwell equations we obtain another scheme that preserves the divergence of $H+M$ but not the norm of the full magnetization neither the energy estimate. On the opposite of the previous scheme it only requires at each step the inversion of one $6 N_{a} N_{b} \times 6 N_{a} N_{b}$ matrix.

Still using splitting methods (Strang splitting), we then develop a new scheme that preserves 
the norm of the full magnetization, the divergence of $H+M$ in the course of time and that fulfills the following energy estimate:

$$
\begin{aligned}
& \frac{1}{2}\left(\left\|H^{n+1}\right\|^{2}+\left\|E^{n+1}\right\|^{2}+\alpha^{-1}\left\|M^{n+1}\right\|^{2}\right) \\
& +d t \gamma\left\|\frac{1}{\varepsilon} M_{0} \wedge H^{n+1 / 2}+\varepsilon M^{n+1 / 2} \wedge H^{n+1 / 2}-\frac{1}{\varepsilon \alpha} M_{0} \wedge M^{n+1 / 2}\right\|^{2} \\
= & \frac{1}{2}\left(\left\|H^{n}\right\|^{2}+\left\|E^{n}\right\|^{2}+\alpha^{-1}\left\|M^{n}\right\|^{2}\right),
\end{aligned}
$$

where $V^{n+1 / 2}=\frac{V^{n+3 / 4}+V^{n+1 / 4}}{2}$. This one requires at each time step the inversion of a $12 N_{a} N_{b} \times 12 N_{a} N_{b}$ matrix and many inversions of $6 N_{a} N_{b} \times 6 N_{a} N_{b}$ matrices.

Finally we compare in Section 4 the three schemes we obtained. Our main goal is to have an effective scheme that gives at least good qualitative results.

This work follows previous studies on the limit model fulfilled by these perturbations: in [3], T. Colin, C. Galusinski and H. Kaper study the case of small mono-dimensional perturbations, i.e. they consider the evolution in (long) time of the shape of the perturbation according to the direction of propagation and prove that it behaves according to a semilinear heat equation. In a recent work [11] we lead the theoretical study of the behaviour of the perturbation in a bi-dimensional case (the direction of propagation and a slow transversal direction): we find that the perturbation $\left(\alpha^{-1 / 2} \widetilde{M}, \widetilde{H}, \widetilde{E}\right)(t)$ remains close for $t \in\left[0, T / \varepsilon^{2}\right]$ to $U^{0}$, solution of the following Khokhlov-Zabolotskaya type equations:

$$
\left\{\begin{array}{l}
U_{0}=\sum_{j=1}^{k} u_{j} \\
\left(\partial_{t}+v_{j} \partial_{x}\right) u_{j}=0 \\
\partial_{x}\left(\partial_{\tau} u_{j}-D_{j} \partial_{x}^{2} u_{j}+B_{j}\left(u_{j}, \partial_{x} u_{j}\right)+F_{j}\left(u_{j}, u_{j}, u_{j}\right)\right)=C_{j} \partial_{y}^{2} u_{j},
\end{array}\right.
$$

where $U_{0}=\left(\alpha^{-1 / 2} M^{0}, H^{0}, E^{0}\right)$ fulfills the polarization condition: $\left(\alpha^{-1} M^{0}-H^{0}\right) \wedge M_{0}=0$.

We expect that this numerical study highlights some properties of Eq. (1.11) particularly the different velocities of the waves.

\section{Energy estimate}

As there is existence of local and regular solutions to the equations (1.6)-(1.7)-(1.8) (for example, $c f[1])$ we perform some energy estimates on the solution:

$$
\begin{aligned}
\frac{1}{2} \frac{d}{d t}\|M\|_{\mathbb{L}^{2}}^{2}+\frac{1}{\varepsilon^{2}}\left[\int _ { \mathbb { R } ^ { 2 } } \left(\left(M_{0} \wedge H\right) \cdot M-\gamma\left(M_{0} \wedge H\right) \cdot\left(M_{0} \wedge M\right)\right.\right. \\
\left.\left.-\alpha^{-1} \gamma\left|M_{0} \wedge M\right|^{2}\right) d x d y\right]-\gamma \int_{\mathbb{R}^{2}}\left(M_{0} \wedge M\right) \cdot(M \wedge H) d x d y=0 . \\
\frac{1}{2} \frac{d}{d t}\|H\|_{\mathbb{L}^{2}}^{2}-\int_{\mathbb{R}^{2}}\left(k \wedge \partial_{x} E+\varepsilon l \wedge \partial_{y} E\right) \cdot H d x d y=-\int_{\mathbb{R}^{2}} \partial_{t} M \cdot H d x d y . \\
\frac{1}{2} \frac{d}{d t}\|E\|_{\mathbb{L}^{2}}^{2}+\int_{\mathbb{R}^{2}}\left(k \wedge \partial_{x} H+\varepsilon l \wedge \partial_{y} H\right) \cdot E d x d y \\
=\frac{1}{2} \frac{d}{d t}\|E\|_{\mathbb{L}^{2}}^{2}+\int_{\mathbb{R}^{2}}\left(k \wedge \partial_{x} E+\varepsilon l \wedge \partial_{y} E\right) \cdot H d x d y \\
=0
\end{aligned}
$$




$$
\begin{aligned}
& \int_{\mathbb{R}^{2}} \partial_{t} M \cdot H= \\
& \frac{1}{\varepsilon^{2}} \int_{\mathbb{R}^{2}}\left[\alpha^{-1}\left(M_{0} \wedge M\right) \cdot H+\gamma\left|M_{0} \wedge H\right|^{2}-\gamma \alpha^{-1}\left(M_{0} \wedge H\right) \cdot\left(M_{0} \wedge M\right)\right] d x d y \\
& +\gamma \int_{\mathbb{R}^{2}}\left[2\left(M_{0} \wedge H\right) \cdot(M \wedge H)-\alpha^{-1}\left(M_{0} \wedge M\right) \cdot(M \wedge H)+\varepsilon^{2}|M \wedge H|^{2}\right] d x d y .
\end{aligned}
$$

Finally, we obtain:

$$
\frac{1}{2} \frac{d}{d t}\left(\|H\|_{\mathbb{L}^{2}}^{2}+\|E\|_{\mathbb{L}^{2}}^{2}+\alpha^{-1}\|M\|_{\mathbb{L}^{2}}^{2}\right)+\gamma\left\|\frac{1}{\varepsilon} M_{0} \wedge H+\varepsilon M \wedge H-\frac{1}{\varepsilon \alpha} M_{0} \wedge M\right\|_{\mathbb{L}^{2}}^{2}=0 .
$$

\section{Numerical Scheme}

In this section we investigate the numerical approximation of the system (1.6)-(1.8) which is made of the Maxwell equations coupled to the Landau-Lifshitz equation.

\subsection{Space Discretisation}

The solutions of the problem propagate at a finite speed ( $c f[3]$ and [11]). The velocities are:

$$
v_{ \pm 1}= \pm\left(\frac{1}{1+\alpha}\right)^{1 / 2}, \quad v_{ \pm 2}= \pm\left(\frac{1+\left(1-\left(k \cdot M_{0}\right)^{2}\right) \alpha}{1+\alpha}\right)^{1 / 2}
$$

We then consider a periodic domain $\Omega=[0, a] \times[0, b]$ instead of $\mathbb{R}^{2}$ with the length $a$ and the width $b$ so that the support of $M, H, E$ does not reach the boundaries of $\Omega$ for the times we consider $\left(t \leq 1 / \varepsilon^{2}\right)$.

We use a classical $N_{a} \times N_{b}$ Yee mesh where the unknown for the magnetic field and the magnetization are taken at the corners and in the middle of the cell. For the electric field we use a translated grid where the unknowns are taken at the middle of the edges of the cell (Figure 1).

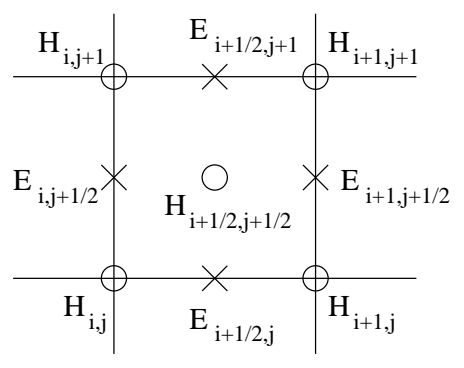

Figure 1: Space discretisation (Yee scheme)

We then express the derivatives in space of $H$ (resp. $E$ ) on the mesh of $E$ (resp. $H$ ). We denote by $D_{x}^{E}$ and $D_{y}^{E}$ (resp. $D_{x}^{H}$ and $D_{y}^{H}$ ) the numerical operator of derivation $\frac{\partial}{\partial x}$ and $\frac{\partial}{\partial y}$ on the 
mesh of $E$ (resp. $H$ ) expressed on the mesh of $H$ (resp. $E)$ :

$$
\begin{array}{ll}
\left(D_{x}^{E} E\right)_{i, j}=\frac{E_{i+1 / 2, j}-E_{i-1 / 2, j}}{\delta x} & \left(D_{x}^{E} E\right)_{i+1 / 2, j+1 / 2}=\frac{E_{i+1, j+1 / 2}-E_{i, j+1 / 2}}{\delta x} \\
\left(D_{y}^{E} E\right)_{i, j}=\frac{E_{i, j+1 / 2}-E_{i, j-1 / 2}}{\delta y} & \left(D_{y}^{E} E\right)_{i+1 / 2, j+1 / 2}=\frac{E_{i+1 / 2, j+1}-E_{i+1 / 2, j}}{\delta y} \\
\left(D_{x}^{H} H\right)_{i+1 / 2, j}=\frac{H_{i+1, j}-H_{i, j}}{\delta x} & \left(D_{x}^{H} H\right)_{i, j+1 / 2}=\frac{H_{i+1 / 2, j+1 / 2}-H_{i-1 / 2, j+1 / 2}}{\delta x} \\
\left(D_{y}^{H} H\right)_{i+1 / 2, j}=\frac{H_{i+1 / 2, j+1 / 2}-H_{i+1 / 2, j-1 / 2}}{\delta y} & \left(D_{y}^{H} H\right)_{i, j+1 / 2}=\frac{H_{i, j+1}-H_{i, j}}{\delta y}
\end{array}
$$

where $\delta x=\frac{a}{N_{a}}$ and $\delta y=\frac{b}{N_{b}}$.

\subsection{Time discretisation}

As the equations (1.6)-(1.7)-(1.8) fulfill an energy estimate, we are looking for a scheme satisfying such properties:

\subsubsection{Semi-implicit scheme}

By considering the general form of the Landau-Lifshitz-Maxwell equations used in [3] and [11], we let:

$$
U^{n}=\left(\alpha^{-1 / 2} M^{n}, H^{n}, E^{n}\right)^{t},
$$

and we use the following discretisation:

$$
\begin{array}{r}
\frac{U^{n+1}-U^{n}}{d t}+A_{x}^{1}\left(\frac{U^{n+1}+U^{n}}{2}\right)+\varepsilon A_{y}^{2}\left(\frac{U^{n+1}+U^{n}}{2}\right)+\frac{1}{\varepsilon^{2}}\left(L_{0}+L_{1}\right)\left(\frac{U^{n+1}+U^{n}}{2}\right) \\
+\left(\begin{array}{ccc}
B_{1}\left(U^{n}\right) & B_{2}\left(U^{n}\right) & 0 \\
B_{3}\left(U^{n}\right) & B_{4}\left(U^{n}\right) & 0 \\
0 & 0 & 0
\end{array}\right)\left(\frac{U^{n+1}+U^{n}}{2}\right)=0,
\end{array}
$$

where

$$
\left\{\begin{aligned}
& A_{x}^{1}=\left(\begin{array}{ccc}
0 & 0 & 0 \\
0 & 0 & -(k \wedge)\left(D_{x}^{H}\right) \\
0 & (k \wedge)\left(D_{x}^{E}\right) & 0
\end{array}\right), \quad A_{y}^{2}=\left(\begin{array}{ccc}
0 & 0 & 0 \\
0 & 0 & -(l \wedge)\left(D_{y}^{H}\right) \\
0 & (l \wedge)\left(D_{y}^{E}\right) & 0
\end{array}\right), \\
& L_{0}(\cdot)=\left(\begin{array}{ccc}
-\alpha^{-1} M_{0} \wedge \cdot \alpha^{-1 / 2} M_{0} \wedge \cdot & 0 \\
\alpha^{-1 / 2} M_{0} \wedge \cdot & -M_{0} \wedge \cdot & 0 \\
0 & 0 & 0
\end{array}\right), \\
& L_{1}(\cdot)=\gamma\left(\begin{array}{ccc}
-\alpha^{-1} M_{0} \wedge\left(M_{0} \wedge \cdot\right) & \alpha^{-1 / 2} M_{0} \wedge\left(M_{0} \wedge \cdot\right) & 0 \\
\alpha^{-1 / 2} M_{0} \wedge\left(M_{0} \wedge \cdot\right) & -M_{0} \wedge\left(M_{0} \wedge \cdot\right) & 0 \\
0 & 0
\end{array}\right), \\
& B_{1}(u) v=v_{1} \wedge u_{2}+\gamma v_{1} \wedge\left(M_{0} \wedge u_{2}\right)-\gamma \alpha^{-1 / 2} v_{1} \wedge\left(M_{0} \wedge u_{1}\right)+\varepsilon^{2} \gamma \alpha^{1 / 2} v_{1} \wedge\left(u_{1} \wedge u_{2}\right), \\
& B_{2}(u) v=\gamma M_{0} \wedge\left(u_{1} \wedge v_{2}\right), \\
& B_{3}(u) v=\gamma u_{1} \wedge\left(M_{0} \wedge v_{1}\right), \\
& B_{4}(u) v=-\alpha^{1 / 2} u_{1} \wedge v_{2}-\gamma \alpha^{1 / 2} M_{0} \wedge\left(u_{1} \wedge v_{2}\right)-\gamma \alpha^{1 / 2} u_{1} \wedge\left(M_{0} \wedge v_{2}\right)-\varepsilon^{2} \alpha \gamma u_{1} \wedge\left(u_{1} \wedge v_{2}\right),
\end{aligned}\right.
$$


with $u=\left(u_{1}, u_{2}, u_{3}\right)^{t}$ and $v=\left(v_{1}, v_{2}, v_{3}\right)^{t}$.

It is a first order scheme in time and second order one in space. Following the proof of the energy estimate (2.1), we have:

$$
\begin{aligned}
& \frac{1}{2}\left(\left\|H^{n+1}\right\|^{2}+\left\|E^{n+1}\right\|^{2}+\alpha^{-1}\left\|M^{n+1}\right\|^{2}\right) \\
& +d t \gamma\left\|\frac{1}{\varepsilon} M_{0} \wedge H^{n+1 / 2}+\varepsilon M^{n} \wedge H^{n+1 / 2}-\frac{1}{\varepsilon \alpha} M_{0} \wedge M^{n+1 / 2}\right\|^{2} \\
& =\frac{1}{2}\left(\left\|H^{n}\right\|^{2}+\left\|E^{n}\right\|^{2}+\alpha^{-1}\left\|M^{n}\right\|^{2}\right),
\end{aligned}
$$

where $H^{n+1 / 2}=\frac{H^{n+1}+H^{n}}{2}$ and $M^{n+1 / 2}=\frac{M^{n+1}+M^{n}}{2}$.

Although this scheme is $\mathbb{L}^{2}$-stable, it does not preserve the norm $\left|M_{0}+\varepsilon^{2} M\right|$ of the magnetization and $\operatorname{div}(H+M)$. It also needs at each step the inversion of a matrix whose size is $18 N_{a} N_{b} \times$ $18 N_{a} N_{b}$ (where $N_{a}$ (resp. $N_{b}$ ) is the number of points in the $x$ (resp. $y$ ) direction). In the following this scheme will be referred to as "SIS" (Semi-Implicit Scheme).

\subsubsection{Splitting}

Another solution consists in the use of a splitting scheme. We then only have to solve separately the Maxwell equations and the Landau-Lifshitz equation.

At each step we solve at first the Maxwell equations using the Yee scheme [12]: we calculate the electric field at times $(n+1 / 2) \delta t$ and the magnetic field and the magnetization vector at times $n \delta t$ :

$$
\left\{\begin{array}{l}
M^{n+1 / 2}=M^{n} \\
E^{n}=E^{n-1 / 2}-\delta t\left(k \wedge D_{x}^{H} H^{n}+\varepsilon l \wedge D_{y}^{H} H^{n}\right) \\
H^{n+1 / 2}=H^{n}+\delta t\left(k \wedge D_{x}^{E} E^{n+1 / 2}+\varepsilon l \wedge D_{y}^{E} E^{n+1 / 2}\right) .
\end{array}\right.
$$

Although the Yee scheme is ill-adapted to obtain an energy estimate in this splitting method it allows us to explicitly obtain the electromagnetic field, thus reducing the numbers of required computations.

We then solve the Landau-Lifshitz equation using a semi-implicit scheme:

$$
\left\{\begin{array}{l}
E^{n+1 / 2}=E^{n}, \\
C=M^{n+1 / 2}+H^{n+1 / 2}=M^{n}+H^{n+1 / 2}, \\
M^{n+1}=M^{n+1 / 2}+\delta t\left(\frac{1}{\varepsilon^{2}} L\left(-C+\left(1+\alpha^{-1}\right) \frac{M^{n+1}+M^{n+1 / 2}}{2}\right)+L^{\prime}\left(\frac{M^{n+1}+M^{n+1 / 2}}{2}\right)\right. \\
\left.+B\left(\frac{M^{n+1}+M n+1 / 2}{2}, M^{n+1 / 2}\right)\right), \\
H^{n+1}=C-M^{n+1},
\end{array}\right.
$$

where

$$
\left\{\begin{array}{l}
L(\cdot)=\left(M_{0} \wedge \cdot\right)+\gamma M_{0} \wedge\left(M_{0} \wedge \cdot\right) \\
L^{\prime}(\cdot)=(C \wedge \cdot)+\gamma M_{0} \wedge(C \wedge \cdot)+\gamma\left(\left(M_{0} \wedge C\right) \wedge \cdot\right), \\
B(U, V)=\gamma U \wedge\left(\left(\left(1+\alpha^{-1}\right) M_{0}+\varepsilon^{2} C\right) \wedge V\right) .
\end{array}\right.
$$

Unfortunately this scheme does not preserve any energy estimate such that the one of Th. (1.1) neither the norm of the full magnetization $M_{0}+\varepsilon^{2} M$. This is due to the way we compute the non-linearity of the Landau-Lifshitz equation (as well as the use of the Yee scheme to compute the Maxwell equations). However we immediatly obtain that $\operatorname{div}\left(H^{n}+M^{n}\right)$ is constant on the course of time and this scheme only requires the inversion at each step of a $6 N_{a} N_{b} \times 6 N_{a} N_{b}$ matrix. This scheme will be referred to as "SpS" (Splitting Scheme). 


\subsubsection{Some numerical improvements: Strang splitting}

Since the two previous schemes have major drawbacks we develop a new one using the Strang splitting in time: we first solve the Maxwell equations on $[t, t+d t / 2]$,

Semi-implicit scheme for the Maxwell equations.

$$
\left\{\begin{array}{l}
M^{n+1 / 4}=M^{n}, \\
\frac{H^{n+1 / 4}-H^{n}}{\delta t / 2}-\left(k \wedge D_{x}^{E} \frac{E^{n+1 / 4}+E^{n}}{2}+\varepsilon l \wedge D_{y}^{E} \frac{E^{n+1 / 4}+E^{n}}{2}\right)=0 \\
\frac{E^{n+1 / 4}-E^{n}}{\delta t / 2}+\left(k \wedge D_{x}^{H} \frac{H^{n+1 / 4}+H^{n}}{2}+\varepsilon l \wedge D_{y}^{H} \frac{H^{n+1 / 4}+H^{n}}{2}\right)=0 .
\end{array}\right.
$$

then we solve the Landau-Lifshitz equation on $[t, t+d t]$,

Scheme for the Landau-Lifshitz equation.

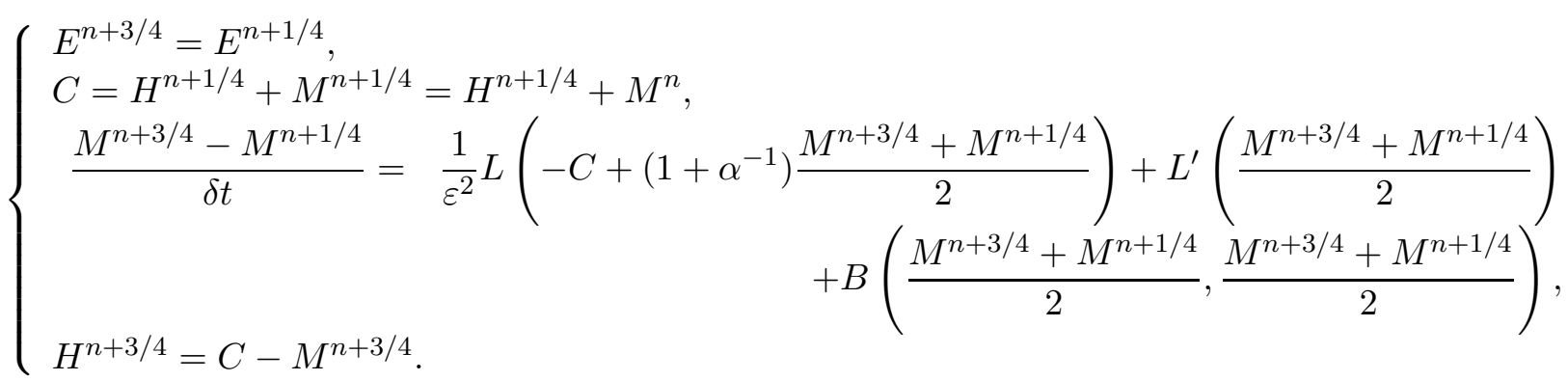

The non-linearity prevents us from obtaining $M^{n+3 / 4}$ implicitly. We get around this difficulty by using a fixed point method:

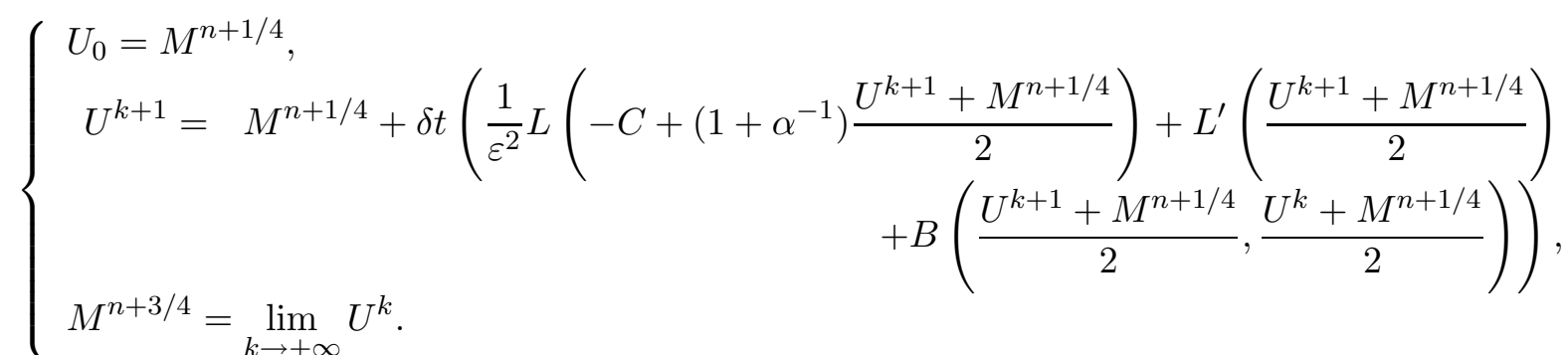

We then reapply the scheme for the Maxwell equations on $[t+d t / 2, t+d t]$ :

$$
\left\{\begin{array}{l}
M^{n+1}=M^{n+3 / 4}, \\
\frac{H^{n+1}-H^{n+3 / 4}}{\delta t / 2}-\left(k \wedge D_{x}^{E} \frac{E^{n+1}+E^{n+3 / 4}}{2}+\varepsilon l \wedge D_{y}^{E} \frac{E^{n+1}+E^{n+3 / 4}}{2}\right)=0, \\
\frac{E^{n+1}-E^{n+3 / 4}}{\delta t / 2}+\left(k \wedge D_{x}^{H} \frac{H^{n+1}+H^{n+3 / 4}}{2}+\varepsilon l \wedge D_{y}^{H} \frac{H^{n+1}+H^{n+3 / 4}}{2}\right)=0 .
\end{array}\right.
$$

For this scheme we obtain immediatly that $\operatorname{div}\left(H^{n}+M^{n}\right)$ does not vary in the course of time and that

$$
\left|M_{0}+\varepsilon^{2} M^{n+1}\right|^{2}=\left|M_{0}+\varepsilon^{2} M^{n}\right|^{2}, \quad \forall n \in \mathbb{N}
$$


We also obtain energy estimates for this scheme:

First Step:

$$
\left\{\begin{array}{l}
M^{n+1 / 4}=M^{n} \\
\frac{1}{2}\left(\left\|E^{n+1 / 4}\right\|^{2}+\left\|H^{n+1 / 4}\right\|^{2}\right)=\frac{1}{2}\left(\left\|E^{n}\right\|^{2}+\left\|H^{n}\right\|^{2}\right) .
\end{array}\right.
$$

\section{Second step:}

$$
\left\{\begin{array}{l}
E^{n+3 / 4}=E^{n+1 / 4} \\
\quad \frac{1}{2}\left(\left\|H^{n+3 / 4}\right\|^{2}+\alpha^{-1}\left\|M^{n+3 / 4}\right\|^{2}\right) \\
\quad+d t \frac{M^{n+3 / 4}-M^{n+1 / 4}}{d t} \cdot\left(C-\left(1+\alpha^{-1}\right) \frac{M^{n+3 / 4}+M^{n+1 / 4}}{2}\right) \\
=\frac{1}{2}\left(\left\|H^{n+1 / 4}\right\|^{2}+\alpha^{-1}\|M n+1 / 4\|^{2}\right),
\end{array}\right.
$$

where $C=H^{n+1 / 4}+M^{n+1 / 4}=H^{n+3 / 4}+M^{n+3 / 4}$ and

$$
\begin{aligned}
& \frac{M^{n+3 / 4}-M^{n+1 / 4}}{d t} \cdot\left(C-\left(1+\alpha^{-1}\right) \frac{M^{n+3 / 4}+M^{n+1 / 4}}{2}\right) \\
= & \gamma\left\|\varepsilon M^{n+1 / 2} \wedge H^{n+1 / 2}+\frac{1}{\varepsilon} M_{0} \wedge H^{n+1 / 2}-\alpha^{-1} M_{0} \wedge M^{n+1 / 2}\right\|^{2},
\end{aligned}
$$

where

$$
M^{n+1 / 2}=\frac{M^{n+3 / 4}+M^{n+1 / 4}}{2}, \quad H^{n+1 / 2}=\frac{M^{n+3 / 4}+M^{n+1 / 4}}{2} .
$$

\section{Third Step:}

$$
\left\{\begin{array}{l}
M^{n+1}=M^{n+3 / 4}, \\
\frac{1}{2}\left(\left\|E^{n+1}\right\|^{2}+\left\|H^{n+1}\right\|^{2}\right)=\frac{1}{2}\left(\left\|E^{n+3 / 4}\right\|^{2}+\left\|H^{n+3 / 4}\right\|^{2}\right) .
\end{array}\right.
$$

Finally,

$$
\begin{aligned}
& \frac{1}{2}\left(\left\|E^{n+1}\right\|^{2}+\left\|H^{n+1}\right\|^{2}+\alpha^{-1}\left\|M^{n+1}\right\|^{2}\right) \\
& +\gamma d t\left\|\varepsilon M^{n+1 / 2} \wedge H^{n+1 / 2}+\frac{1}{\varepsilon} M_{0} \wedge H^{n+1 / 2}-\alpha^{-1} M_{0} \wedge M^{n+1 / 2}\right\|^{2} \\
& =\frac{1}{2}\left(\left\|E^{n}\right\|^{2}+\left\|H^{n}\right\|^{2}+\alpha^{-1}\left\|M^{n}\right\|^{2}\right) .
\end{aligned}
$$

This scheme fulfills then the same type of energy estimates we prove on the continuous equation. It requires at each step the inversion of two matrix whose size are $12 N_{a} N_{b} \times 12 N_{a} N_{b}$ (Maxwell equations) and the inversion at each step of the fixed point method of a $6 N_{a} N_{b} \times 6 N_{a} N_{b}$ matrix. In the following this scheme will be referred to as "ECSpS" (Energy Conservating Splitting Scheme)

\section{Numerical simulations}

As explained in the introduction we are interested in the behaviour of small perturbations of the steady-state solutions of the Maxwell-Landau-Lifshitz equations. These solutions are given by:

$$
M_{0}=\left(\begin{array}{c}
\cos \varphi_{0} \\
\sin \varphi_{0} \\
0
\end{array}\right), \quad H_{0}=\frac{1}{\alpha}\left(\begin{array}{c}
\cos \varphi_{0} \\
\sin \varphi_{0} \\
0
\end{array}\right), \quad E_{0}=\left(\begin{array}{l}
0 \\
0 \\
0
\end{array}\right)
$$


for some $\left.\varphi_{0} \in\right] 0, \pi[$. At $t=0$, we perturb this basic solution near the origin. The perturbation depends on two space parameters who varies along the axes defined by the vector $\widetilde{k}$ and $\widetilde{l}$ :

$$
\widetilde{k}=\left(\begin{array}{l}
1 \\
0 \\
0
\end{array}\right), \quad \tilde{l}=\left(\begin{array}{l}
0 \\
0 \\
1
\end{array}\right)
$$

The perturbation is uniform in the third direction defined by $\widetilde{k} \wedge \widetilde{l}$ and is given by

$$
M(0, x, y)=\frac{1}{\varepsilon^{2}}\left[\left(\begin{array}{c}
\cos \left(\varphi_{0}+\varepsilon^{2} \varphi\right) \cos \left(\varepsilon^{2} \psi\right) \\
\sin \left(\varphi_{0}+\varepsilon^{2} \varphi\right) \cos \left(\varepsilon^{2} \psi\right) \\
\sin \left(\varepsilon^{2} \psi\right)
\end{array}\right)-\left(\begin{array}{c}
\cos \varphi_{0} \\
\sin \varphi_{0} \\
0
\end{array}\right),\right],
$$

where $\varphi$ et $\psi$ are sharply peaked near the center of the domain $[0, a] \times[0, b]$ in $x$ and $y$, where $x$ and $y$ are the coordinates along the axes defined respectively by $\widetilde{k}$ and $\widetilde{l}$ :

$$
\varphi=\psi=e^{-4\left(X^{2}+Y^{2}\right)}
$$

where $X=x-a / 2$ and $Y=y-b / 2$.

The initial perturbation for $H$ and $E$ is also taken to be $\mathcal{O}(1)$ with the additional condition that the initial data fulfills the polarization condition given in [11]:

$$
\left(\alpha^{-1} M(0, x, y)-H(0, x, y)\right) \wedge M_{0}=0 .
$$

All computations reported in this section refer to the case $\varepsilon^{2}=0.01, \gamma=1$ and $\alpha=2$.

\subsection{Comparison between the schemes}

In this section we take:

$$
\varphi=\frac{\pi}{6}, \quad H(t=0, x, y)=\alpha^{-1} M(t=0, x, y)+f M_{0}, \quad E_{0}^{0}=0, \quad f=2 .
$$

Our main objective consists in highlighting the differences and the similarities between these schemes. In a first time we only compare them in the $1 D$ case to obtain a first validation, we then look at them in the $2 D$ case.

\subsubsection{Comparison between the $1 D$ schemes}

In a first attempt to compare these methods, we consider the $1 D$-case, i.e. the case where the initial data is uniform along the $y$-axis (directed by $\widetilde{l}$ ). As the three schemes SIS, ECSpS and SpS use the same discretizations for the derivatives in space and as they preserve the monodimensional feature of the solution (we show this conservation only for one scheme (ECSpS) Fig. 2) we are lead back to the case studied by Colin, Galusinski and Kaper in [3].

We consider the evolution in time of the energy given by the three schemes (Fig 3).

As the schemes SIS and ECSpS theoretically fulfill energy estimates (respectively (3.1) and (3.2)), we look numerically at their accuracy and plot:

$$
\frac{1}{2}\left(\left\|H^{n}\right\|^{2}+\left\|E^{n}\right\|^{2}+\left\|M^{n}\right\|^{2}\right)+\gamma d t \sum_{k=1}^{n}\left\|D^{k}\right\|^{2},
$$

(Fig. 4) where, 

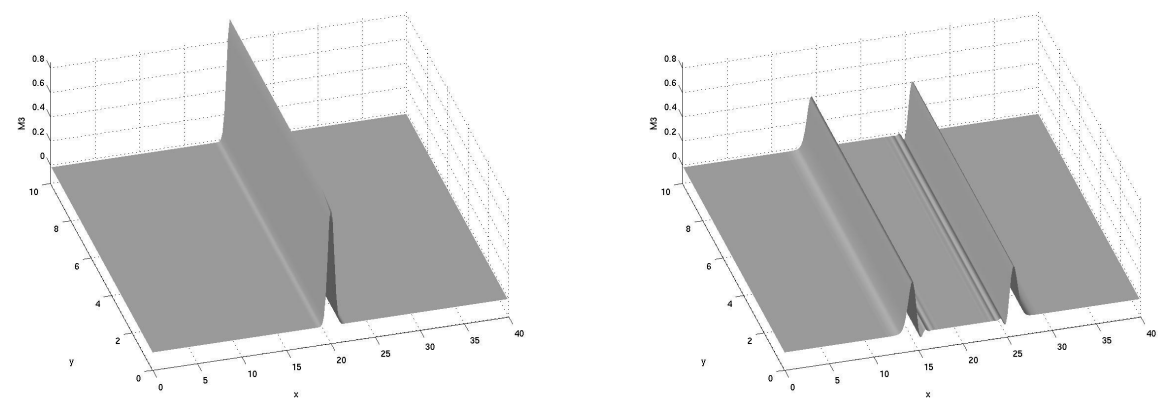

$t=0: 1 D$ initial data for $M_{3}$

$t=10: M_{3}$ still monodimensional

Figure 2: Conservation of the monodimensional feature for SpS.

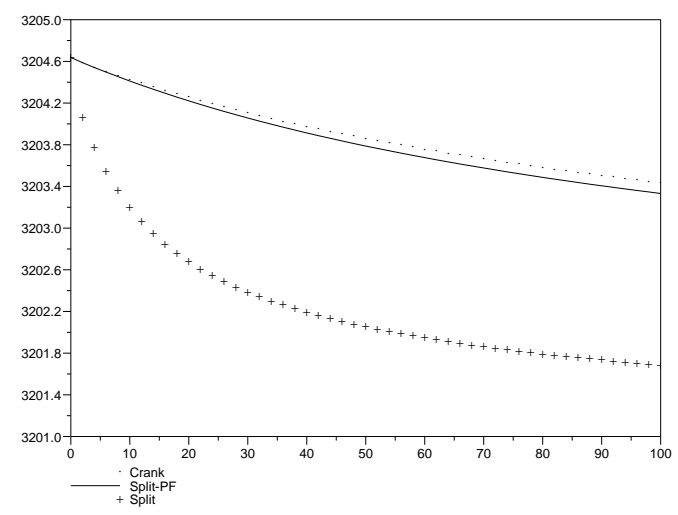

Figure 3: Evolution of the energy in the course of time until $\frac{T}{\varepsilon^{2}}$. 
- for the SIS scheme:

$$
D^{k}=\frac{1}{\varepsilon} M_{0} \wedge \frac{H^{k-1}+H^{k}}{2}+\varepsilon M^{k-1} \wedge \frac{H^{k-1}+H^{k}}{2}-\frac{1}{\varepsilon \alpha} M_{0} \wedge \frac{M^{k-1}+M^{k}}{2},
$$

- for the SpS and the ECSpS scheme:

$$
D^{k}=\frac{1}{\varepsilon} M_{0} \wedge \frac{H^{k-1}+H^{k}}{2}+\varepsilon \frac{M^{k-1}+M^{k}}{2} \wedge \frac{H^{k-1}+H^{k}}{2}-\frac{1}{\varepsilon \alpha} M_{0} \wedge \frac{M^{k-1}+M^{k}}{2},
$$

and where $E^{k}=\frac{E^{k-1 / 2}+E^{k+1 / 2}}{2}$ for the SpS scheme.

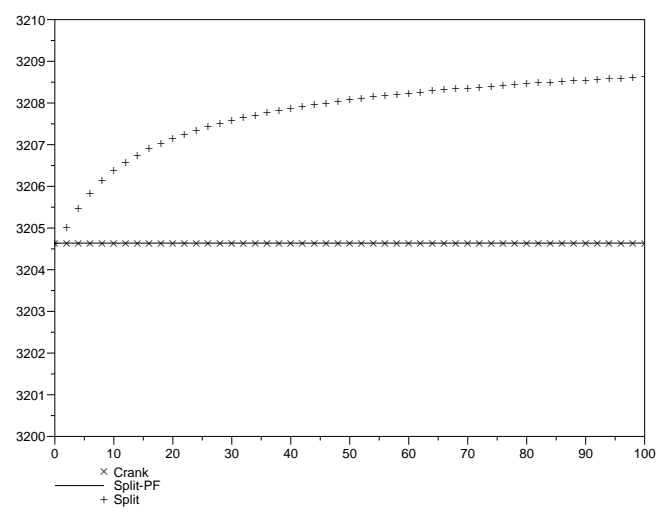

Figure 4: Numerical evolution of the theoretical conservation law in the course of time until $\frac{T}{\varepsilon^{2}}$.

Even if the scheme SpS does not fulfill the same energy estimates as ECSpS and SIS we observe that the energy for SpS remains close from the one for SIS and ECSpS. More precisely we consider the evolution in time of the relative difference on the energy, particularly when the discretization steps are halved (Fig. 5).

We observe numerical convergence for the three schemes as the discretisations steps go to zero. We note that the norm of the full magnetization $M_{0}+\varepsilon M$ is preserved by ECSpS. We then plot the maximum and the minimum of $\left|M_{0}+\varepsilon^{2} M\right|^{2}$ in the course of time (Fig. 6).

The last point we have to verify is the preservation of $\operatorname{div}(H+M)$. We then plot $\| \operatorname{div}(H+$ $M)-\operatorname{div}(H+M)_{t=0} \|_{\mathbb{L}_{\infty}}($ Figure 7$)$ :

As we are considering waves, we now show a component of the magnetization at various times (Figure 8). We observe the propagation of the data in the course of time at the same velocity for the three schemes. The high oscillations are purely numerical effects that disappear when we refine the discretization in SIS and ECSpS.

\subsubsection{Comparison between the $2 D$ schemes}

At first, let us consider a small transversal perturbation of a $1 D$ solution:

$$
\varphi=\psi=e^{-4 X^{2}}\left(1+\varepsilon^{2} e^{-4 Y^{2}}\right) M_{0}^{0} .
$$




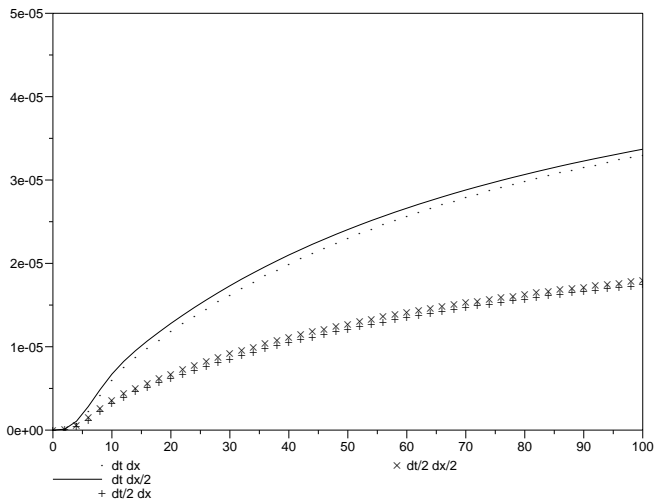

Difference between SIS and ECSpS

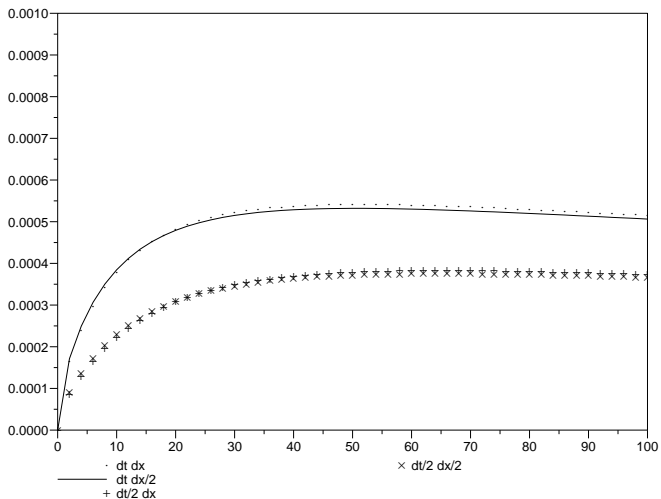

Difference between ECSpS and SpS

Figure 5: Relative difference between the energies of the 1D schemes until $\frac{T}{\varepsilon^{2}}$.

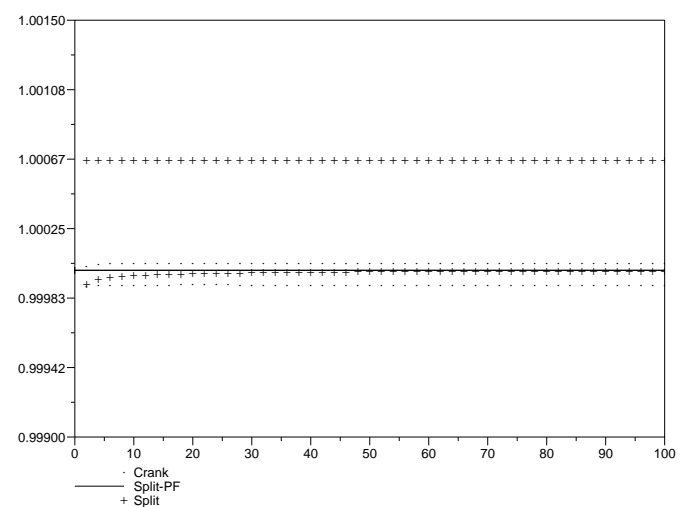

Figure 6: Evolution of the norm of $M_{0}+\varepsilon^{2} M$ in the course of time until $\frac{T}{\varepsilon^{2}}$.

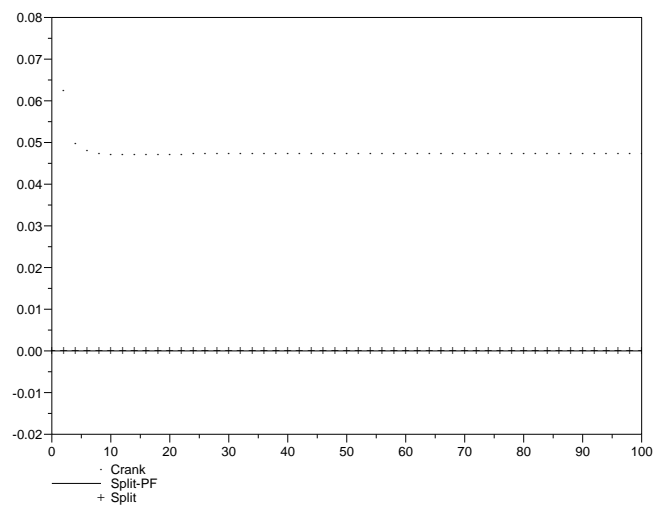

Figure 7: Evolution of $\operatorname{div}(H+M)$ in the course of time until $\frac{T}{\varepsilon^{2}}$. 


$$
\text { At } \frac{T}{5 \varepsilon^{2}}
$$

SIS
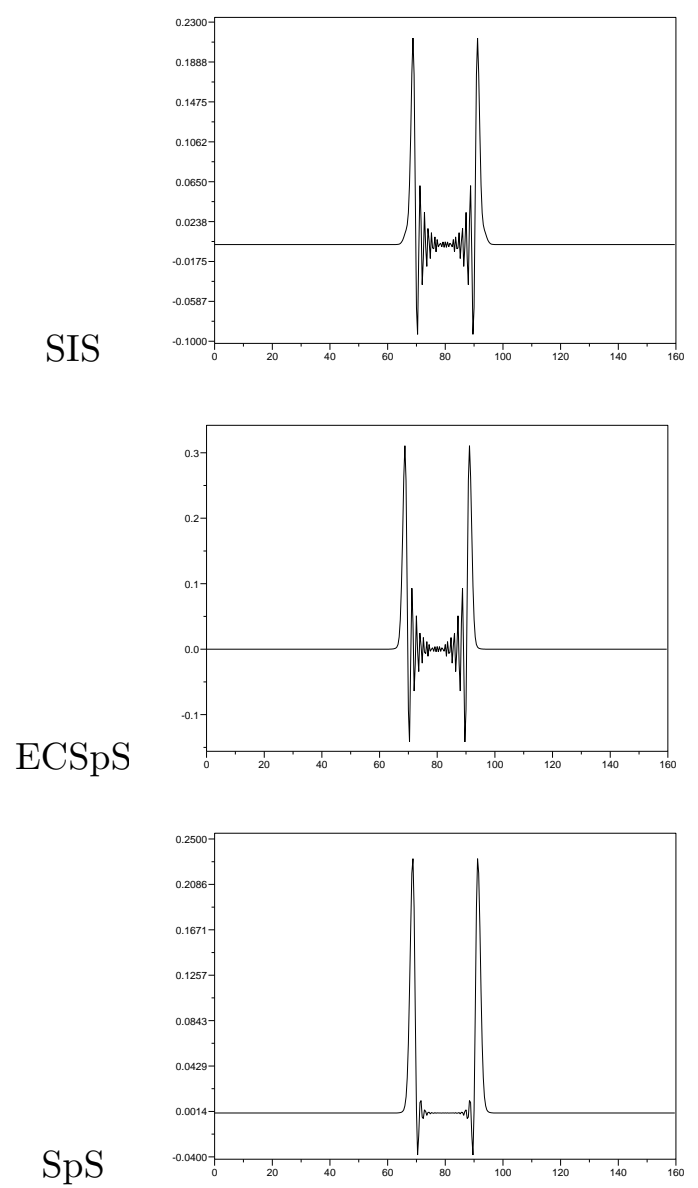

At $\frac{T}{\varepsilon^{2}}$
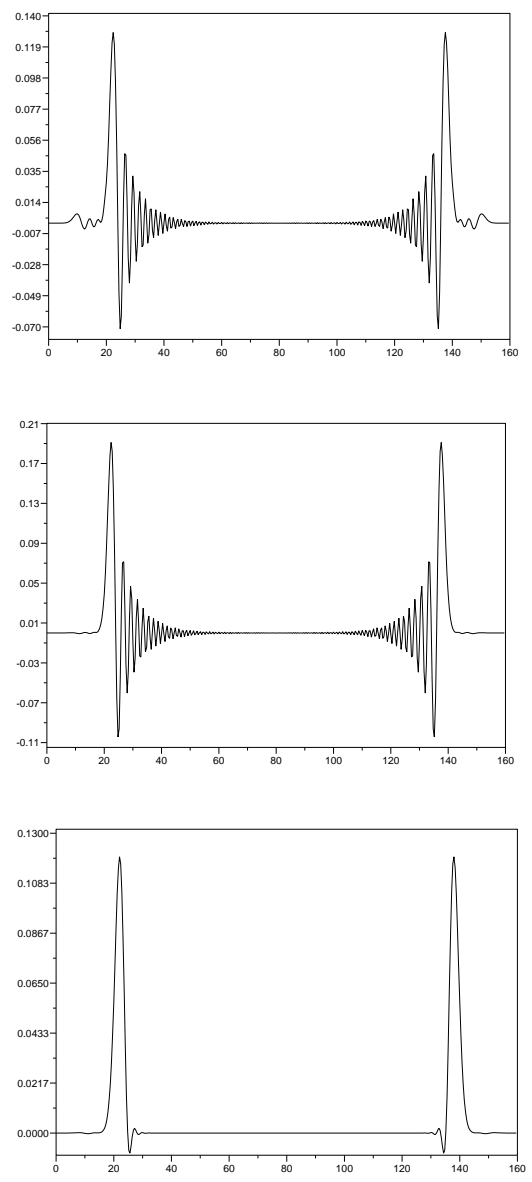

Figure 8: $M_{3}$ at various times for SIS, ECSpS and SpS. 
To observe the effect of this small perturbation, we consider the difference between the solution obtained with the previous initial data and the unperturbed $1 D$ initial data (Figure 9). We observe a $2 D$ behaviour of the perturbation.

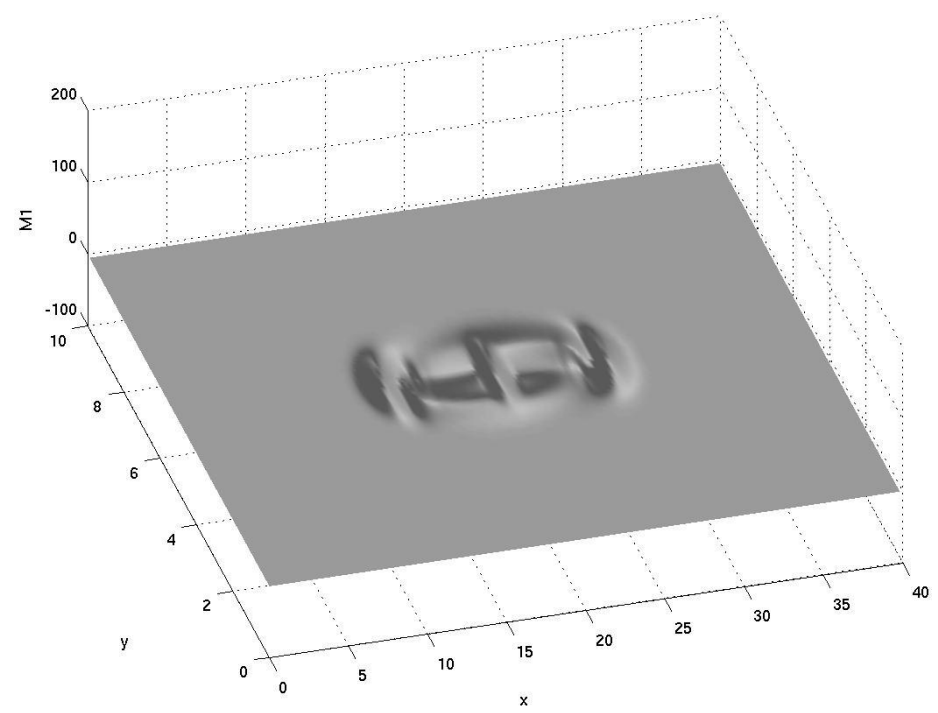

Figure 9: Perturbation of the $1 D$ case for $M_{1}$ at $\frac{T}{\varepsilon}$ (ECSpS scheme).

We now compare the three schemes in the $2 D$ case. As the schemes SIS and ECSpS need a lot more calculations than SpS, we reduce the time interval on which we compare them. This also allows us to reduce the space domain of study since the informations propagate at a finite speed. As in the $1 D$ case, we verify that the energy evolves according to the estimates we obtained for SIS and ECSpS (Figure 10).

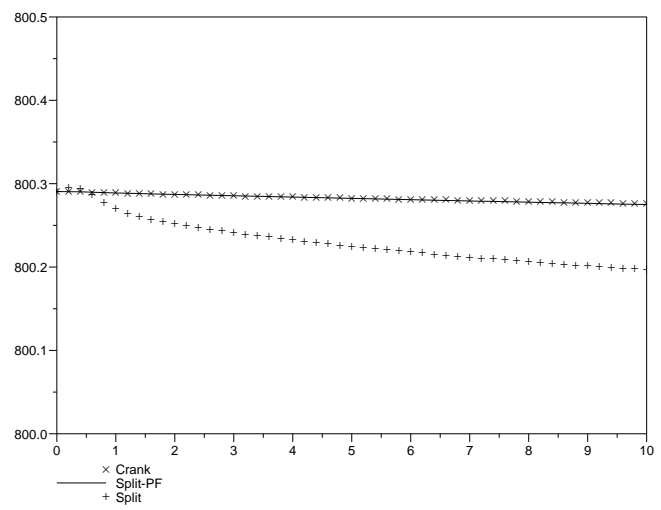

Figure 10: Evolution of the energy in the course of time until $\frac{T}{\varepsilon}$. 
Since the schemes SIS and ECSpS respectively fulfill the conservation laws (3.1) and (3.2), we expect to observe them numerically (Figure 11) and we plot as for the $1 D$ case :

$$
\frac{1}{2}\left(\left\|H^{n}\right\|^{2}+\left\|E^{n}\right\|^{2}+\left\|M^{n}\right\|^{2}\right)+\gamma d t \sum_{k=1}^{n}\left\|D^{k}\right\|^{2},
$$

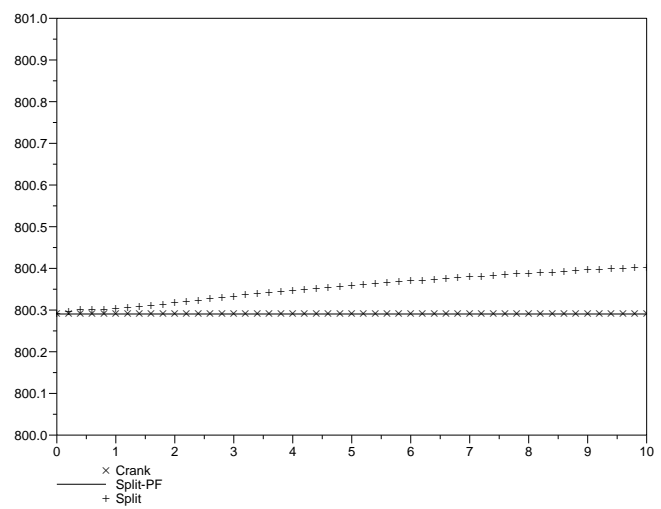

Figure 11: Numerical evolution of the theoretical conservation law in the course of time until $\frac{T}{\varepsilon}$.

We observe now the evolution in time of the relative difference between the energies when the discretization steps are halved (Figure 12).

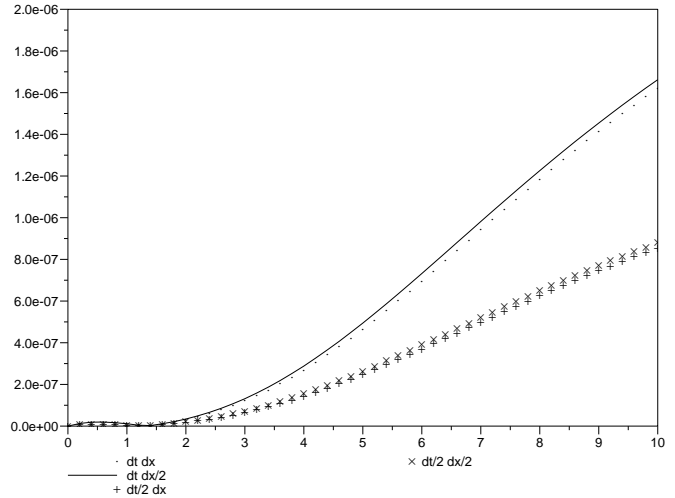

Difference between SIS and ECSpS

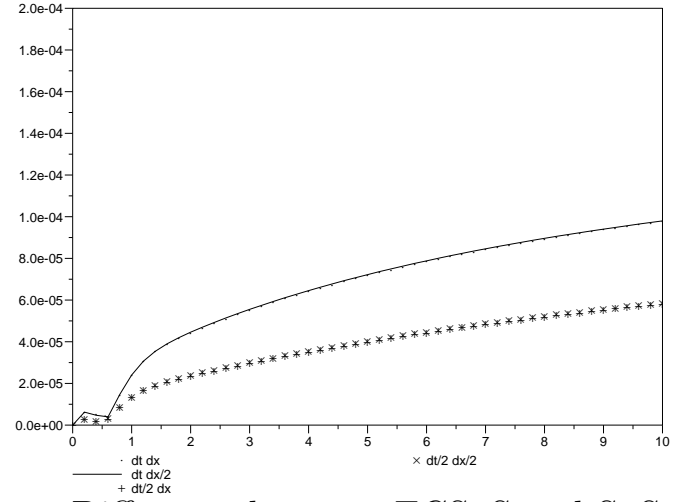

Difference between ECSpS and SpS

Figure 12: Relative difference between the energies of the $2 \mathrm{D}$ schemes until $\frac{T}{\varepsilon}$.

Remark 4.1. On Figure 10 we remark that the energy for SpS grows initially then decreases. 
This problem seems inherent to $S p S$ and purely numerical and we can see on Figure 12 that this error decreases with the time step.

As the figure 12 suggests it, the three schemes give energetically consistant results and they converge toward the same solution as the discretization steps go to zero. We now look at the evolution of the maximum and the minimum of the norm of the full magnetization $M_{0}+\varepsilon^{2} M$ (Figure 13).

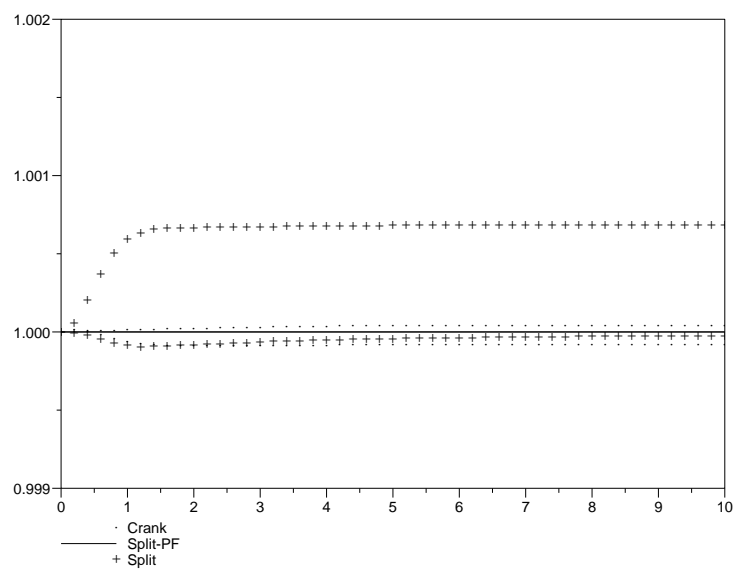

Figure 13: Evolution of the norm of $M_{0}+\varepsilon^{2} M$ in the course of time until $\frac{T}{\varepsilon}$.

And we also look at the preservation of $\operatorname{div}(H+M)$. We then plot $\| \operatorname{div}(H+M)-\operatorname{div}(H+$ $M)_{t=0} \|_{\mathbb{L}^{\infty}}$ (Figure 14):

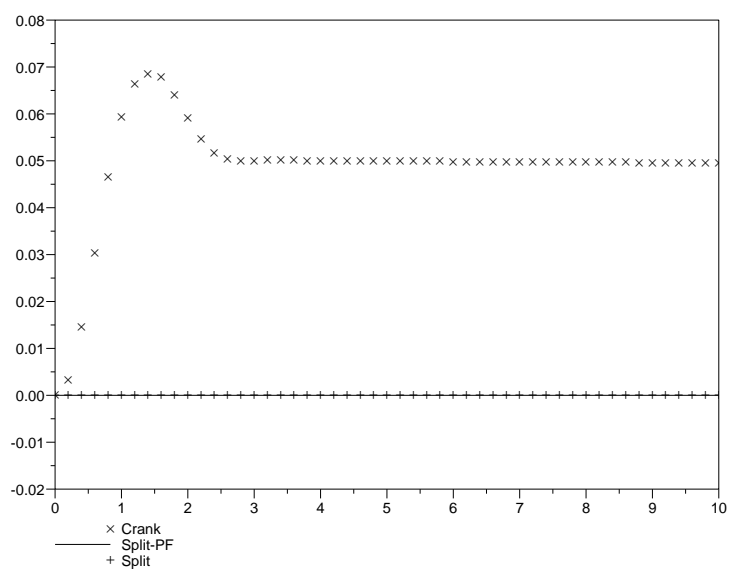

Figure 14: Evolution of $\operatorname{div}(H+M)$ in the course of time until $\frac{T}{\varepsilon}$.

We now compare visually the obtained solutions at $\frac{T}{\varepsilon}$ (Figure 15$)$.

As in the mono-dimensional case we observe that the peaks appear at the same points, the informations propagate then at the same speed. 
SIS
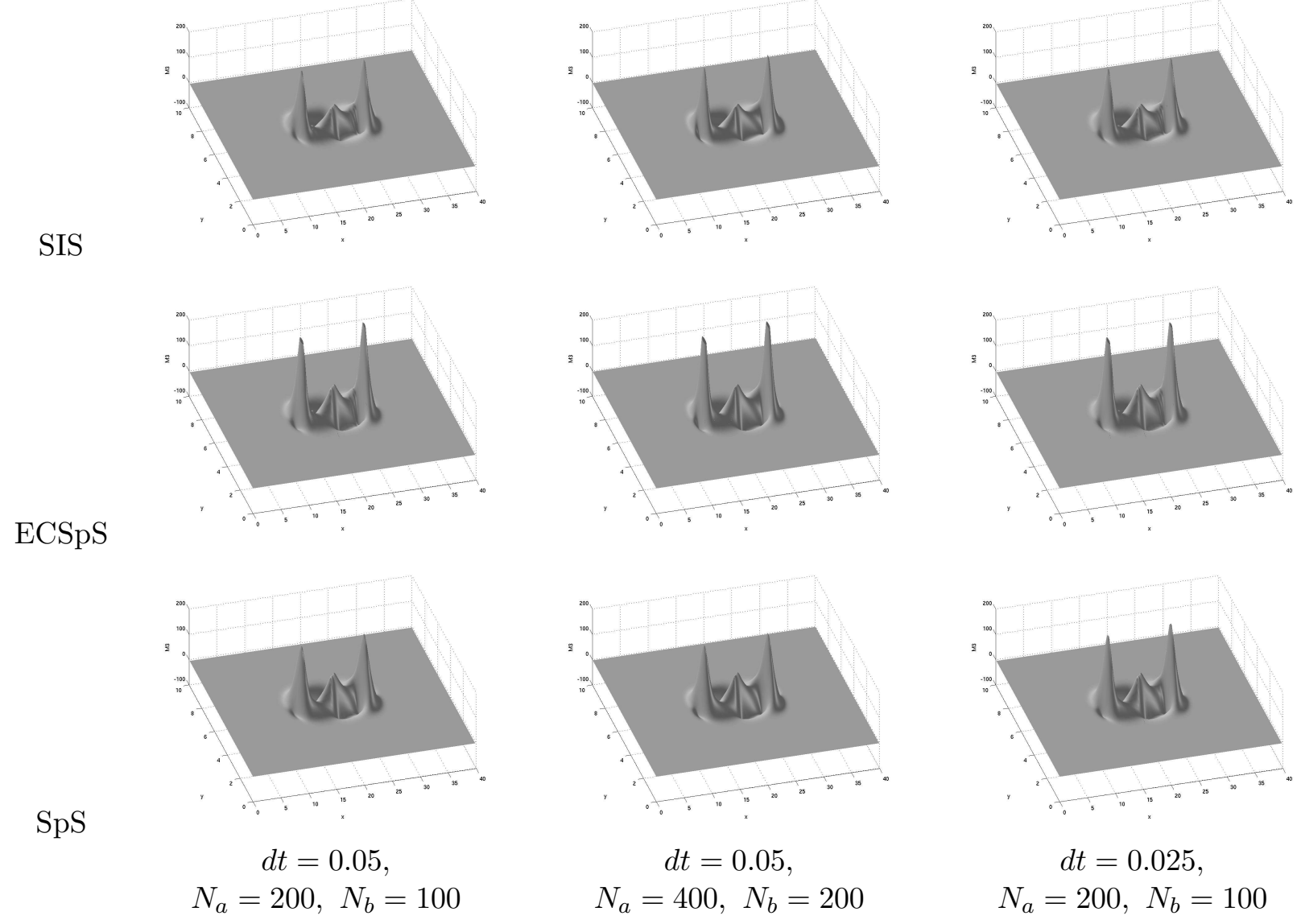

Figure 15: $M_{3}$ at $\frac{T}{\varepsilon}$. 
The three schemes give comparable qualitative results. Moreover, even if we have no theoretical stability results on the scheme SpS neither the preservation of the norm for the full magnetization with SpS and SIS, these schemes are numerically stable and converge toward the solution given by ECSpS as the discretisation steps tend to zero and give the good qualitative behaviour of the solution.

In the following we will use ECSpS since he fulfills more properties.

\subsection{Velocities and influence of $\varphi$}

In [11] we prove that the solution splits in five standing and traveling waves. The eigenvectors $\left(M_{0}^{i}, H_{0}^{i}, E_{0}^{i}\right)$ associated to the non-null velocities $v_{i}, i= \pm 1, \pm 2$ are:

$$
\begin{array}{ll}
M_{0}^{ \pm 1}= \pm \alpha k \wedge M_{0}, & M_{0}^{ \pm 2}=\mp \alpha \cos \varphi M_{0} \wedge\left(k \wedge M_{0}\right), \\
H_{0}^{ \pm 1}= \pm k \wedge M_{0}, & H_{0}^{ \pm 2}=\mp \cos \varphi M_{0} \wedge\left(k \wedge M_{0}\right), \\
E_{0}^{ \pm 1}=\sqrt{1+\alpha} k \wedge\left(k \wedge M_{0}\right), & E_{0}^{ \pm 2}=\frac{1}{\cos \varphi} \sqrt{1+\alpha} \sqrt{1+\alpha \sin ^{2} \varphi} k \wedge M_{0} .
\end{array}
$$

To highlight the different velocities, we use ECSpS with $\varphi=\frac{\pi}{6}$ and $\varphi=\frac{\pi}{3}$ (Figure 16).
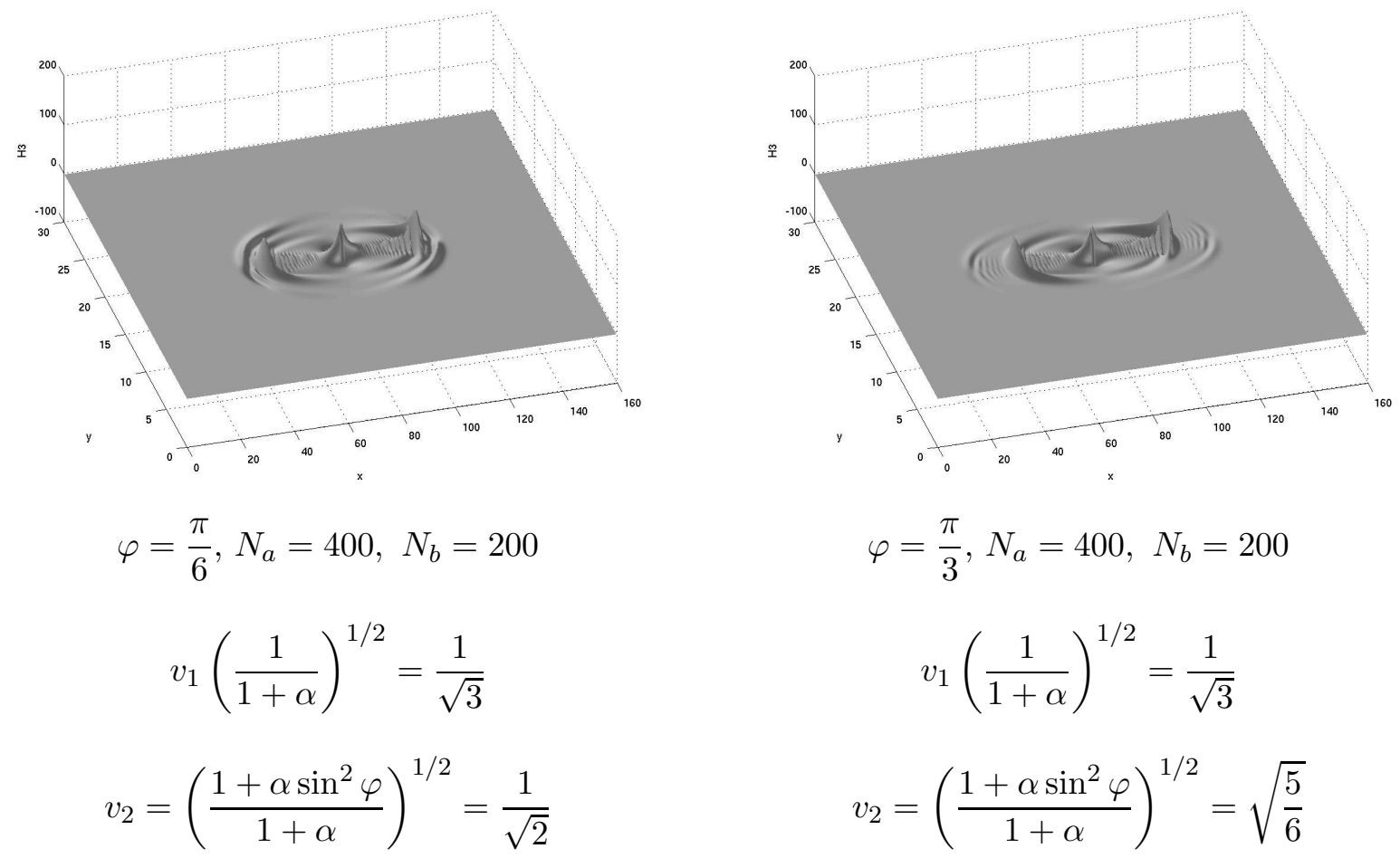

$$
\begin{gathered}
\varphi=\frac{\pi}{3}, N_{a}=400, N_{b}=200 \\
v_{1}\left(\frac{1}{1+\alpha}\right)^{1 / 2}=\frac{1}{\sqrt{3}} \\
v_{2}=\left(\frac{1+\alpha \sin ^{2} \varphi}{1+\alpha}\right)^{1 / 2}=\sqrt{\frac{5}{6}}
\end{gathered}
$$

Figure 16: $H_{3}$ at $\frac{T}{2 \varepsilon^{2}}$.

We observe the expected difference between the velocities: as the initial data is placed at the center of the domain we find a ratio close to $\sqrt{\frac{3}{2}} \simeq 1.225$ for $\varphi=\frac{\pi}{6}$ and a ratio close to $\sqrt{\frac{5}{2}} \simeq 1.581$. As on the previous figures we also observe the diffraction of the perturbation in 
the transverse direction, and that according to the Khokhlov-Zabolotskaya equation which rules the main profile.

We verify in this case that the numerical scheme agrees with the equations fulfilled by the profile and we observe the expected velocities for the propagation.

We moreover observe a lot of small ripples. This effect is purely numerical and tends to disappear when we double the number of mesh used (Figure 17).
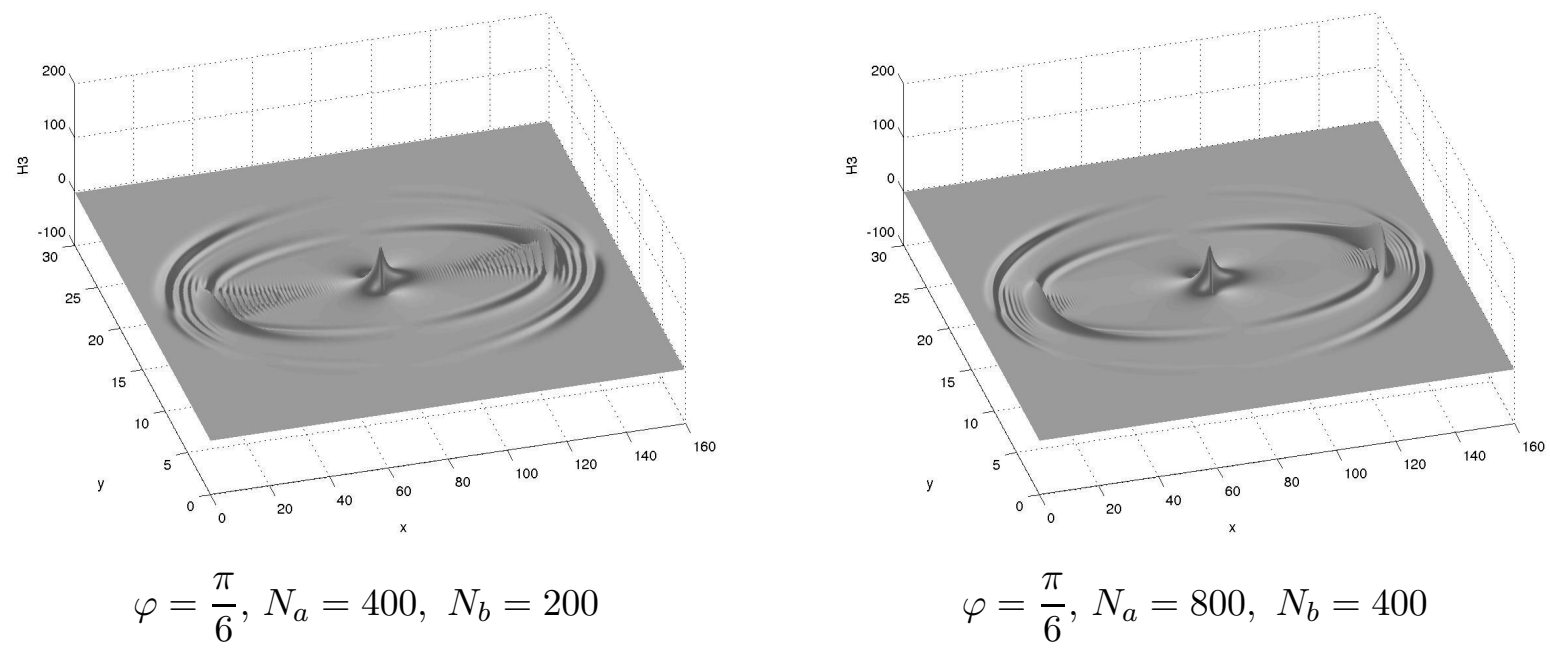

Figure 17: $H_{3}$ at $\frac{T}{\varepsilon^{2}}$.

Acknowledgments: The author wishes to thank his Ph. D. advisors G. Carbou and P. Fabrie for their help. He is also grateful to T. Boucherès, C. Galusinski and H. Leblond for fruitful discussions.

\section{References}

[1] S. Alinhac, P. Gerard, Opérateurs pseudo-différentiels et opérateurs de Nash-Moser, Savoirs actuels, InterEditions/Editions du CNRS,1991.

[2] G. Carbou, P. Fabrie, O. Guès, On the ferromagnetism equations in the non static case, Commun. Pure Appl. Anal. 3, No.3, 367-393 (2004).

[3] T. Colin, C. Galusinski, H. Kaper, Waves in ferromagnetic media, Commun. Partial Differ. Equations 27, No.7-8, 1625-1658 (2002).

[4] T.L. Gilbert, A Lagrangian formulation of gyromagnetic equation of the magnetization field, Phys. Rev. 100 (1955) 1243.

[5] L. Halpern and S. Labbé, .

[6] L. Landau and E. Lifshitz, On the theory of magnetic permeability in ferromagnetic body, Physik. Z. Soviet Union 8 (1935) 153-169. 
[7] H. Leblond, private communication.

[8] H. Leblond, M. Manna, Coalescence of electromagnetic travelling waves in a saturated ferrite, J. Phys. A: Math. Gen. 26 (1993), 6451-6468.

[9] P.B. Monk and O. Vacus, Error estimates for a numerical scheme for ferromagnetic problems, SIAM J. Numer. Anal. 36 (1999), No. 3, 696-718.

[10] J. Necas, Les méthodes directes en théorie des équations elliptiques, Masson, 1967.

[11] D. Sanchez, Long waves in ferromagnetic media, Zabolotskaya-Khokhlov equation, J. Differential Equations 210 (2005), No. 2, 263-289.

[12] K.S. Yee, Numerical Solution of Initial Boundary Value Problems Involving Maxwell's Equations in Isotropic Media, IEEE Trans. Antennas Propag. 14, 302 (1966), 302-307.

[13] E.A. Zabolotskaya, R.V. Khokhlov, Quasi-planes waves in the nonlinear acoustics of confined beams, Sov. Phys. Acoust 8, 35. 